\title{
Shaky life of a water drop in an anise oil-rich environment
}

\author{
Óscar R. Enríquez $\odot,{ }^{1},{ }^{*}$ Daniel Robles, ${ }^{2}$ Pablo Peñas-López, ${ }^{3}$ and Javier Rodríguez-Rodríguez ${ }^{1}$ \\ ${ }^{1}$ Fluid Mechanics Group, Carlos III University of Madrid, 28911 Leganés, Madrid, Spain \\ ${ }^{2}$ Centro de Ciencias de la Atmósfera, Universidad Nacional Autónoma de México, México DF, México \\ ${ }^{3}$ Physics of Fluids Group, University of Twente, Enschede, The Netherlands
}

(Received 4 February 2019; published 24 October 2019)

\begin{abstract}
This paper is associated with a video winner of a 2018 APS/DFD Milton van Dyke Award for work presented at the DFD Gallery of Fluid Motion. The original video is available online at the Gallery of Fluid Motion, https://doi.org/10.1103/APS.DFD.2018. GFM.V0054
\end{abstract}

DOI: 10.1103/PhysRevFluids.4.100502

We illustrate the rich physical phenomena that can arise in certain kinds of three-liquid mixtures. In this particular case, we use anethole (anise oil), ethanol, and water. The situation can be humorously compared to a "love triangle" enacted by fluids: anethole and ethanol are miscible (they "love" each other), and so are water and ethanol; however, water and anethole are only sparingly soluble (they "hate" each other). Put the three together, and ...

The best-known example of this type of mix is termed the ouzo effect and is seen upon adding water to anise-based alcoholic drinks (ouzo, pastis, raki, anís, arak, absinthe, etc.), which triggers the emulsification of anise oil, giving these drinks their characteristic milky aspect. However, this is interesting not only for enjoying drinks but also in exploring spontaneous solvent separation processes, the formation of nanodroplets, microemulsions, and more.

A multicomponent drop dissolving in another multicomponent host liquid displays a wealth of phenomena occurring in interface physics: diffusion-driven mass transfer, capillary forces, Marangoni stresses, rich contact-line dynamics, buoyancy-driven flows, etc. Besides being interesting due to all the involved physics, this kind of flow finds many applications in modern microchemistry processes, such as solvent extraction [1,2].

We explore the fate of a water droplet deposited at the bottom of a mixture of trans-anethole (anise oil) and ethanol when there is at least twice as much of the former as the latter. In contrast to the very slow dissolution of a water drop in pure anethole or the fast spreading and mixing in alcohol, in this case droplets oscillate and grow. Oscillations start shortly after deposition, presumably due to Marangoni stresses that arise as the liquids' compositions along the interface start to change spatially. This composition change also triggers a gravity current downwards and away from the drop along the bottom, which can be seen in Figs. 1(a)-1(c) [indicated by the arrow in Fig. 1(a)]. Note that, to make the droplets more apparent and to help understand the mixing process, the drops have been dyed with blue ink. Since $\rho_{\mathrm{H}_{2} \mathrm{O}} \approx \rho_{\text {anethole }}>\rho_{\text {ethanol }}$, this gravity current suggests that ethanol is preferentially dissolving into the droplet, making this current rich in (heavier) anethole. That this is indeed the case becomes clear as the droplet grows and deforms due to buoyancy while

*oenrique@ing.uc3m.es

Published by the American Physical Society under the terms of the Creative Commons Attribution 4.0 International license. Further distribution of this work must maintain attribution to the author(s) and the published article's title, journal citation, and DOI. 
(a)

(b)
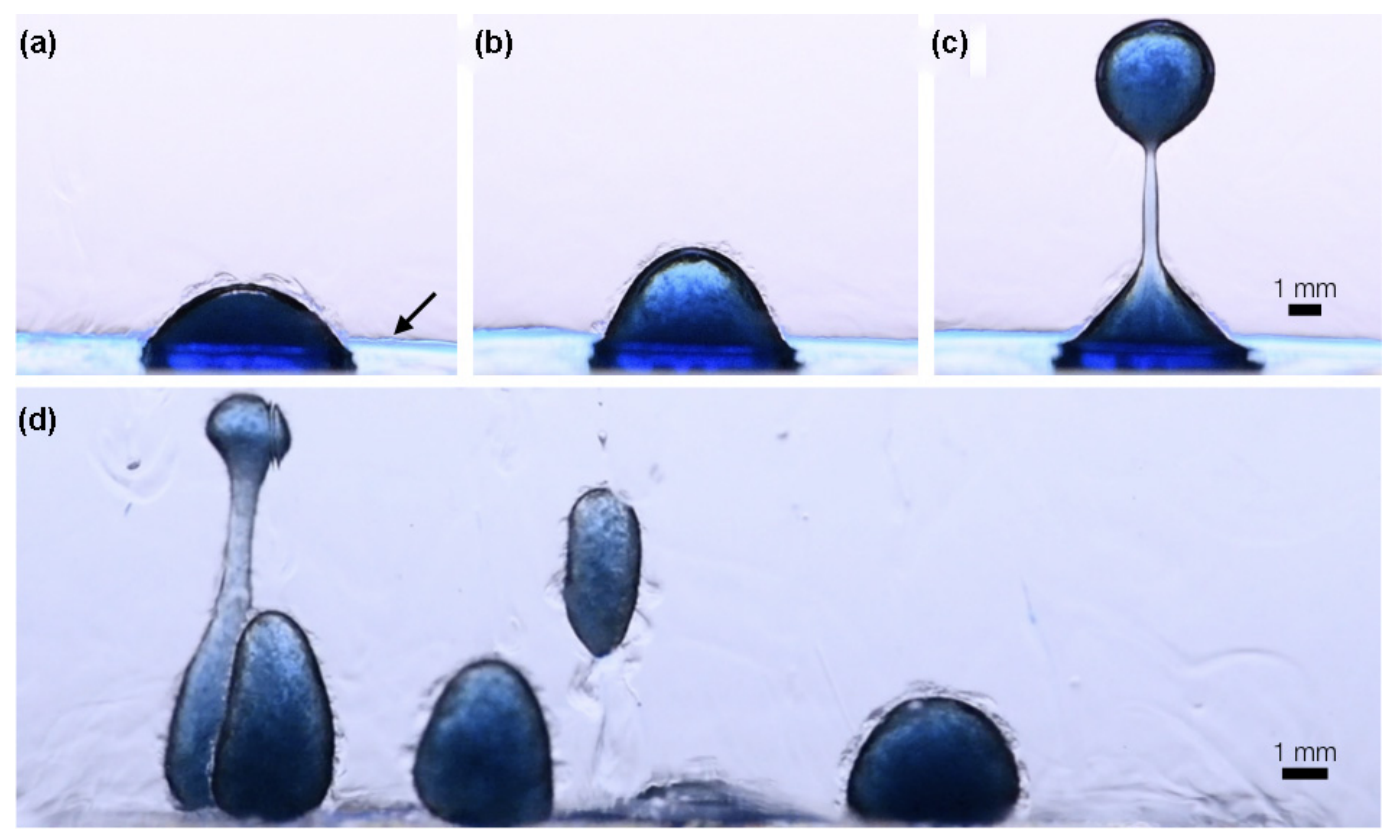

FIG. 1. (a)-(c) Evolution of a water droplet (dyed blue for contrast) upon being deposited in a 5:1 (volume) anethole:ethanol solution. Time after deposition: (a) $33 \mathrm{~s}$, (b) $63 \mathrm{~s}$, and (c) $69 \mathrm{~s}$. (d) Several water droplets $8 \mathrm{~s}$ after deposition in a 2:1 anethole:ethanol mixture. https://doi.org/10.1103/APS.DFD.2018.GFM.V0054

remaining pinned to the bottom [Fig. 1(b)], which indicates a reduction in its average density. This reduction is not uniform, as can be inferred from Fig. 2(a), where the top part of the drop is seen to be more clear than the bottom part, indicating that the entrained, undyed fluid is mostly concentrated near the top. Despite this compositional gradient and the flow motion taking place both inside and outside the drop, its shape seems to be well predicted by the equations of hydrostatics, assuming a constant composition [3] [see Fig. 2(a)]. To fit the hydrostatic solution to the drop shape, only

(a)

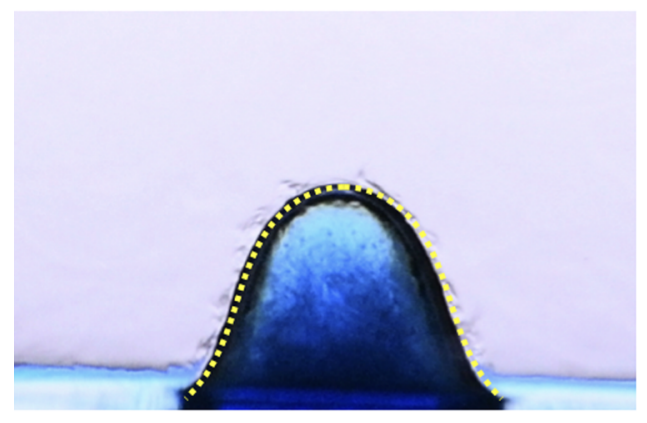

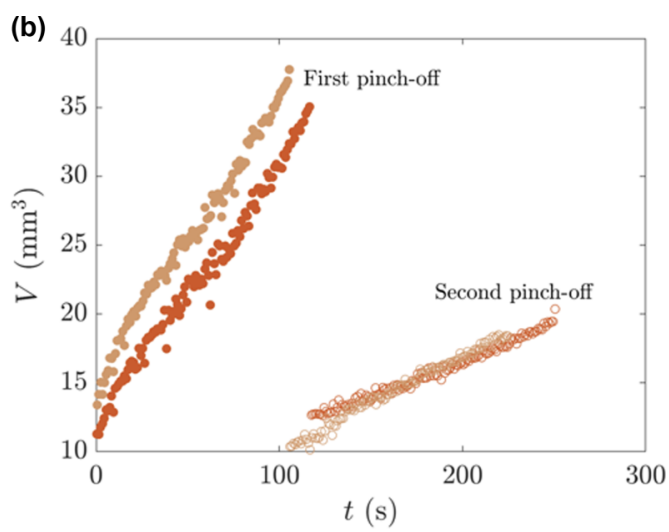

FIG. 2. (a) A drop in a 5:1 solution, 2 s before detachment. The yellow line is the best-fitting hydrostatic solution obtained by adjusting the value of the Bond number, which is Bo $=2.53$ in this case. (b) Time history of droplet volume for two experimental realizations in a 6:1 anethole:ethanol solution. In each case, the first two detachment events are shown. It becomes apparent that the mixing process is much slower after the pinch-off event. 
one free parameter is adjusted, namely, a Bond number defined as Bo $=\Delta \rho g R_{0}^{2} / \sigma$, with $R_{0}$ being the radius at the base of the drop, $g$ being the gravity, $\Delta \rho$ being the density difference between the drop and the host liquid, and $\sigma$ being the interfacial tension. Notice that these last two parameters are actually unknown, so the Bond number obtained through this fitting sets already a relation between them. At later times close to detachment, there is also a noticeable accumulation of anise oil microdroplets inside the water drop, a phenomenon recently characterized by Tan and coworkers [2,4].

Eventually, the growth in volume and the reduction in the average density make the droplet detach, and a new cycle begins. However, as can be seen in Fig. 2(b), the mass transfer process becomes much slower after a pinch-off event. This is a consequence of the liquid left in the drop having a composition closer to that of the host solution than the initial drop. This process goes on for several pinch-off events, as can be seen in the accompanying video, although for the sake of clarity, only two events are shown in Fig. 2.

In the near future we plan to investigate the effect of the initial composition in the flow features. For instance, increasing the ethanol concentration accelerates this series of events [see Fig. 1(d)] and makes the flow more distorted.

We acknowledge the support of the Spanish FEDER/Ministerio de Ciencia, Innovación y Universidades - Agencia Estatal de Investigación through Grants No. DPI2014-59292-C3-1-P, No. DPI2015-71901-REDT, and No. DPI2017-88201-C3-3-R.

[1] D. Lohse, Towards controlled liquid-liquid micro-extraction, J. Fluid Mech. 804, 1 (2016).

[2] H. Tan, Evaporation and dissolution of droplets in ternary systems, Ph.D. thesis, University of Twente, 2018.

[3] L. D. Landau and E. M. Lifshitz, Fluid Mechanics, 2nd ed. (Elsevier, The Netherlands, 2013).

[4] H. Tan, C. Diddens, A. A. Mohammed, J. Li, M. Versluis, X. Zhang, and D. Lohse, Microdroplet nucleation by dissolution of a multicomponent drop in a host liquid, J. Fluid Mech. 870, 217 (2019). 